\title{
NOUVELLE
}

\section{La pensée à fleur de peau}

Michael Schubert, Hector Escriva, Vincent Laudet

\section{Laboratoire de biologie \\ moléculaire de la cellule, CNRS UMR 5161/Inra UMR 1237, École Normale Supérieure de Lyon, 69364 Lyon, France.}

vincent.laudet@ens-lyon.fr
> Le système nerveux central (SNC) est organisé de façon différente chez les deux groupes majeurs d'animaux, les protostomiens (les insectes, les annélides, les mollusques, etc.) et les deutérostomiens (les échinodermes, les chordés, etc.) (Figure 1). D’une façon schématique, chez les protostomiens, le SNC est condensé et situé ventralement et, chez les deutérostomiens, il est condensé et situé dorsalement. Un troisième type d'organisation du système nerveux existe chez les animaux placés par la plupart des analyses phylogénétiques à la base de tous les animaux bilatériens: les cnidaires (les anémones, les coraux, etc.) (Figure 1). Chez ces der- niers, le système nerveux n'est pas central, mais il est structuré sous la forme d'un réseau situé à la base de l'épiderme. Les biologistes de l'évolution se posent ainsi depuis longtemps des questions sur les caractéristiques du système nerveux chez l'ancêtre de tous les animaux bilatériens. Ce débat a donné lieu à une demi-douzaine d'hypothèses différentes. L'objet de cet article n'est pas de faire un récit détaillé des hypothèses existantes; cependant, on peut citer les deux hypothèses les plus représentatives: I'hypothèse d'inversion de Geoffroy-Saint Hilaire (revue plus tard par D. Arendt et K. Nübler-Jung [1]), qui

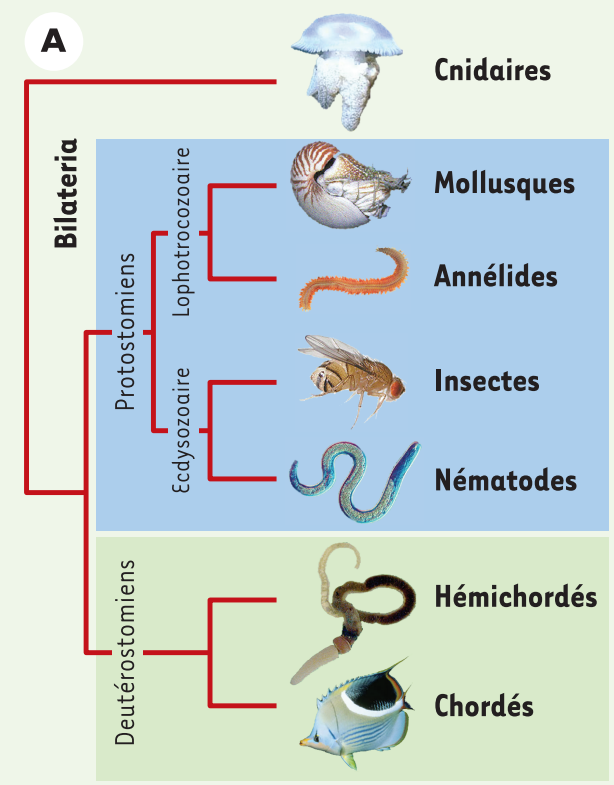

B

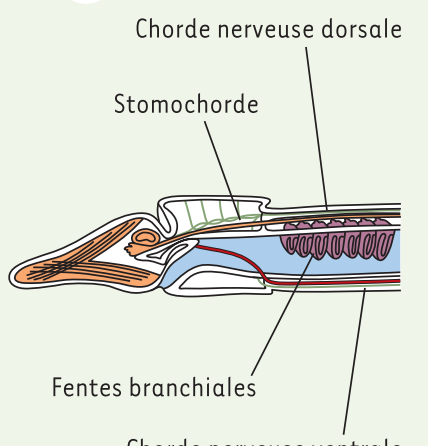

Chorde nerveuse ventrale

Figure 1. Relations phylogénétiques des animaux et morphologie schématique des hémichordés. $A$. Représentation de la phylogénie des principaux groupes de métazoaires. $B$. Représentation schématique de la partie antérieure d'un hémichordé, montrant les deux chordes nerveuses, dorsale et ventrale, en vert, la stomochorde en rouge et les fentes branchiales en violet.

propose un ancêtre commun des protostomiens et deutérostomiens avec un SNC concentré et ventral qui aurait été inversé dorso-ventralement chez les deutérostomiens; et l'hypothèse d'une évolution indépendante chez les protostomiens et les deutérostomiens par condensation ventrale ou dorsale, respectivement, du système nerveux diffus présent chez un ancêtre des animaux bilatériens [2].

Une approche intéressante dans l'étude de l'évolution d'une structure telle que le SNC est l'étude comparative, dans différents modèles animaux, des profils d'expression des gènes impliqués dans le développement de cette structure. II est clair aujourd'hui que l'expression d'un grand nombre de gènes impliqués dans le développement du SNC montre des profils d'expression conservés entre les protostomiens et les deutérostomiens. Mais cette conservation des profils d'expression ne nous permet pas d'inférer une morphologie du SNC commune avec celle d'un ancêtre des animaux bilatériens. Dans un article récent, C.J. Lowe et al. [3] ont utilisé une approche très intéressante pour essayer d'élucider cette question. Ils ont étudié l'expression de vingt-deux gènes chez un hémichordé, Saccoglossus kowalevskii. Ces gènes connus pour être impliqués dans l'organisation spatiale du SNC chez les bilatériens, et très conservés au cours de l'évolution, montrent des profils d'expression comparables entre les insectes et les vertébrés.

Les hémichordés et les échinodermes (oursin, étoile de mer, etc.) sont les deux groupes de deutérostomiens non chordés. Au contraire des échinodermes, les hémichordés montrent plusieurs ressemblances morphologiques avec les chordés, ce qui les rend très intéressants 
comme modèles pour comprendre la mise en place des caractères spécifiques des chordés. Les ressemblances les plus importantes entre les hémichordés et les chordés sont l'existence de fentes branchiales, une stomochorde (assimilée à la notochorde des chordés), et une extension postanale (assimilée à la queue). Leur système nerveux (sans cerveau) est composé d'un réseau de nerfs basi-épithéliaux autour du corps concentré dans deux chordes nerveuses, l'une ventrale et l'autre dorsale, qui ont été proposées par différents auteurs comme étant toutes les deux des homologues de la chorde nerveuse dorsale des chordés (Figure 1). Les résultats de C.J. Lowe montrent une distribution antéro-postérieure similaire des profils d'expression des vingt-deux gènes utilisés entre Saccoglossus et les vertébrés. Mais le résultat le plus surprenant de ce travail n'est pas la distribution antéro-postérieure des gènes étudiés, mais leur distribution dorso-ventrale. En effet, l'expression des gènes étudiés n'est pas concentrée dans la chorde dorsale ou dans la chorde ventrale de Saccoglossus, mais elle se situe au contraire dans plusieurs domaines qui entourent le corps de l'animal, dans le tissu épidermique. Le parallélisme antéro-postérieur des domaines d'expression entre les insectes, les vertébrés, et Saccoglossus, conforte l'hypothèse selon laquelle le rôle de ces gènes dans le contrôle antéro-postérieur du développement du système nerveux est antérieur à la divergence insectes-vertébrés. En ce qui concerne la condensation ventrale ou dorsale du système nerveux, deux possibilités évolutives existent. Elle a pu se produire indépendamment au cours de l'évolution chez les protostomiens et les deutérostomiens respectivement à partir d'un ancêtre possédant un système nerveux diffus; autre possibilité, l'ancêtre des animaux protostomiens et deutérostomiens possédait un système nerveux concentré ventralement, qui a été inversé chez les deutérostomiens, et qui a été perdu secondairement chez les hémichordés [4]. C.J. Lowe et al. préfèrent la première hypothèse et s'interrogent plus sur le mécanisme de la concentration du système nerveux que sur celui de l'inversion de l'axe dorsoventral. Les auteurs se fondent entre autres considérations sur le fait que les phylums placés à la base des animaux bilatériens (les cnidaires et les cténophores) possèdent un système nerveux diffus, et aussi sur le fait que d'autres groupes d'animaux bilatériens (dont la position phylogénétique est controversée) comme les chaetognates, possèdent eux aussi des systèmes nerveux diffus.

De nombreuses questions restent encore non élucidées concernant l'histoire évolutive du système nerveux des animaux. Cependant, le travail de C.J. Lowe et al. montrant un système nerveux diffus et «à fleur de peau» chez les hémichordés, enrichit le débat concernant l'évolution du système nerveux et montre l'utilité des modèles «non classiques» pour les études de biologie évolutive et comparée. Nous attendons donc avec impatience de nouvelles données qui éclairciront quelques-unes de nos questions sur ce vieux sujet qui a été aujourd'hui fortement ravivé. $\diamond$

Thinking with a sensitive skin

\section{RéFÉRENCES}

1. Arendt D, Nubler-Jung K. Inversion of dorsoventral axis? Nature 1994; $371: 26$.

2. Brusca RC, Brusca GJ. Invertebrates, $2^{\text {nd }}$ ed. Sunderland, MA: Siauer, 2003.

3. Lowe CJ, Wu M, Salic A, et al. Anteroposterior patterning in hemichordates and the origins of the chordate nervous system. Cell 2003; 113: 853-65.

4. Holland ND. Early central nervous system evolution: an era of skin brains? Nat Rev Neurosci 2003; 4: 617-27.

\section{NOUVELLE}

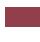 \\ L'opsine en liberté, une des causes de l'amaurose congénitale de Leber}

Simone Gilgenkrantz
9 , rue basse,

54330 Clerey-sur-Brenon,

France.

s.gilgenkrantz@chu-nancy.fr
> Décrite en 1869 par Theodor Leber, l'amaurose congénitale (LCA) est une entité clinique caractérisée par un déficit visuel, diagnostiqué à la naissance ou dans les premiers mois de la vie, et transmis, dans la quasi-totalité des cas, de façon récessive autosomique. Elle représente au moins $5 \%$ des dystrophies rétiniennes et constitue l'une des principales causes de cécité chez l'enfant [1]. L'extinction de l'électrorétinogramme atteste de l'atteinte des deux types de récepteurs: cônes et bâtonnets. Elle peut accompagner des désordres systémiques (syndromes de Joubert, de LhermitteDuclos, de Refsum, et de Zellweger, entre autres). Dans sa forme non syndromique, la LCA regroupe un ensemble de dystro- 